\title{
Cloning and Construction of Overexpression Vector for FaUVR8 Gene Transformation with Strawberry
}

\author{
Xiran Wang ${ }^{1, a}$, Leiyu Jiang ${ }^{1, b}$ and Haoru Tang ${ }^{1, c}$ \\ ${ }^{1}$ Horticulture College, Sichuan Agriculture University, Chengdu 611130, China. \\ a631763272@qq.com, b1291471200@qq.com, chtang@sicau.edu.cn
}

\begin{abstract}
Keywords: Strawberry UVR8 Overexpression Vector construction
Abstract. Anthocyanin is an important production of horticulture plant secondary metabolism, which is tightly concerns with fruit quality. There are lots of factors regulate plant anthocyanin biosynthesis including UV, which is one of the most significant factors, however, the effect mechanism details of UV are still remained to be study. Thus, through DNA cloning technology, total RNA was extracted from the tender leaves of strawberry 'Toyonaka', according to the conserved regions of FaUVR8 from GeneBank (KU647690). The objective cDNA bands with 1410bp were obtained by RT-PCR. Re-combined vector had been constructed using PCR production and the pMDC vector via double-digestion. The result of this recombined vector showed that it was strawberry overexpression vector pMDC-FaUVR8 via vector PCR and vector double-digestion. The construction of this vector would be a basis for further investigation of UVR8 and Agrobacterium-mediated transformations.
\end{abstract}

\section{Introduction}

Strawberry (Fragaria $x$ ananassa Duch.) is Rosaceae (Rosacea) Strawberry (Fragaria) perennial herb, its cultivation area and production of grape berries in the second, in the second, with high economic value. Strawberry is one of the plant models of flavonoids metabolism in plants, and its fruit is rich in anthocyanin [1]. Anthocyanin (Anthocyanin) is the product of flavonoids metabolism pathway in plants. It is not only related to the color of fruit, seed, flower and leaf of higher plants, but also has physiological function to resist biotic and abiotic stresses in plants. Medical research shows that anthocyanins have antioxidant, anticancer, remove the body of free radicals, reducing antioxidant activity, enhance immunity, anti-tumor and protect the gastric mucosa function of [2], it has very broad application prospect.

Studies have shown that the synthesis of anthocyanin in plants is affected by Ultraviolet (UV) radiation, especially ultraviolet B (UV-B) (wavelength: $290-315 \mathrm{~nm}$ ). For plants, UV-B radiation is an important environmental signal, it will affect the metabolism of plants, development process and survival ability and a series of physiological reaction [3]. In early 1996, people from the molecular point of view to explain the effect of UV-B on the model plant, later studies have confirmed that UV-B increased the plant environmental stress, effect of plant morphogenesis, and even affect the vegetation structure. In addition, UV-B also affects the secondary metabolites (e.g. flavonoids, tannins and lignin) in plants which have important physiological functions. Found in the study of photoreceptors on UV-B signaling transduction in UV-B specific receptors in plants is UVR8 (UV RESISTANCE LOCUS 8)[4], UVR8 can be used as a UV sensor protein can dominate plants through a complex series of protective reaction of under UV-B radiation. The response of the corresponding response of UVR8 to UV-B with red and blue light in phytochrome and cryptochrome is similar to but different UVR8 by tryptophan itself color group to feel UV-B. UVR8 in Arabidopsis thaliana is a UV-B specific signal component that mediates the formation of UV-B photosensitive morphogenesis. Studies have shown that UV-B induces the gene expression of encoding UVR8 transcription factor, and it has been proved that UVR8 protein accumulates in the nucleus of plants under the condition of increasing UV-B radiation. Further studies have shown that the UVR8 gene not only participates in the regulation of gene expression in the response to UV protection, but also controls the growth and morphogenesis of plant leaves. UVR8 proved by experiments and optical transduction (ELONGATED HYPOCOTYL 5) HY5 switch closely, and HY5 directly with the anthocyanin 
synthesis of a series of related, so it can be speculated that UVR8 biosynthesis and accumulation of anthocyanins are linked to. On the relationship between existing UVR8 and anthocyanin accumulation test through genetic transformation pathway in Cucumber, apple, horticultural crops such as Populus expression were studied, but the mechanism of UVR8 in strawberry and other perennial herbaceous crops remains unclear, whether with the horticultural plants the same mechanism needs further research.

Overexpression or overexpression refers to the integration of the full-length sequence of the target gene with the highly active constitutive promoter or tissue-specific promoter, and the plant [5] accumulated by this gene product is obtained by transformation technique. Over expression of technology and gene knockout technology is a powerful tool to validate the gene function, but overexpression of gene technology is influenced by functional redundancy is not, therefore overexpression can identify many transcription factor gene knock-out to identify the function of is widely used in the model plant Arabidopsis now. Therefore, strengthening the study of UVR8 has important theoretical significance in regulating anthocyanin content in strawberry and improving the intrinsic quality of strawberry. At the same time, clarifying the roles of UVR8 in response to light in using cultivation measures suitable for facilities (such as light regulation) has important guiding significance to improve the quality of strawberry. Therefore, the overexpression vector of strawberry FaUVR8 gene was constructed, which laid the foundation for the identification of gene function by Agrobacterium tumefaciens infecting plants.

\section{Result and Analysis}

Extraction of total RNA from Strawberry Leaves. Leaf total RNA extraction and concentration after detection by $1 \%$ agarose gel electrophoresis (Fig. 1A) to detect the integrity of the $28 \mathrm{~S}$ and $18 \mathrm{~S}$ bands were clearly visible, the brightness ratio is approximately 2:1, and no obvious DNA bands showed that the total RNA extracted with good results, can be used for the next experiment.

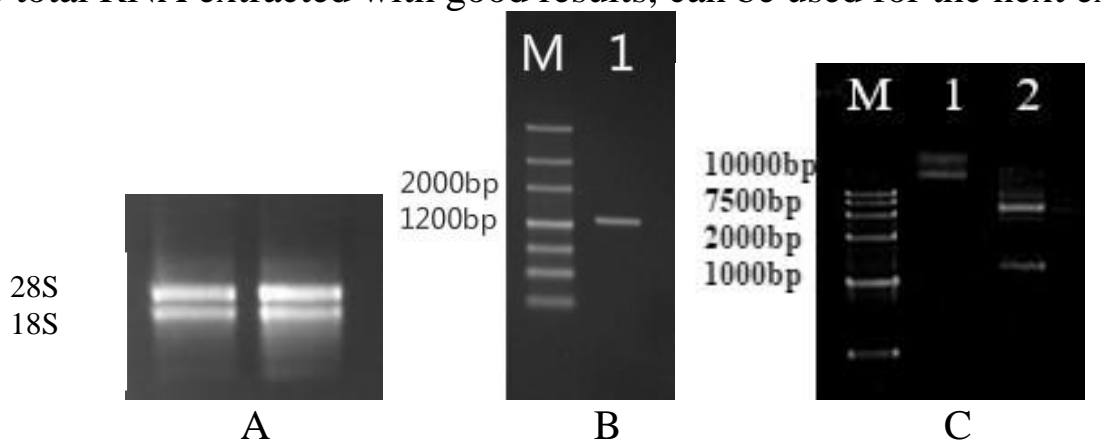

Fig 1. A: Total RNA in strawberry leaves; B: FaUVR8 gene (M: Maker III 1: FaUVR8) ; C: Identification of recombinant plasmid by double enzyme digestion (M: Maker DL15000 1: p MDCFaUVR8 2: pMDC- FaUVR8 Sal I-HF and Kpn I-HF double enzyme digestion).

PCR amplification of FaUVR8 gene. According to the sequence of FaUVR8 GeneBank landing (KU647690) primers were designed to RNA strawberry leaves in reverse transcription cDNA as a template for PCR amplification products by $1 \%$ agarose gel electrophoresis (Fig. 1B). The results showed that the amplified band length is about $1400 \mathrm{bp}$, consistent with the expected results (1406bp), further sequencing analysis showed that the amplified product was the expected target fragment, the sequence is correct, no base mutation.

Construction of overexpression vector of pMDC- FaUVR8. Fragment of PCR amplification products and pMDC vector were confirmed by Sal I-HF and Kpn I-HF double digestion to connect, connect the product of heat shock transformation E.Coli JM109, selected monoclonal colony was extracted plasmid PCR, while the use of Sal I-HF and Kpn I-HF double enzyme digestion (Fig. 1C). The results showed that the bands amplified by PCR were consistent with the expectation, and double enzyme digestion showed that the target fragment had been successfully accessed. 


\section{Materials and Methods}

Materials. The experimental material was a good growing variety of Strawberry (Fragaria $x$ ananassa cv. Toyonaka), which was cultivated in Sichuan Agricultural University. PVPP, 75\% ethanol, chloroform, phenol, acetic acid, isopropyl alcohol, DEPC water treatment; reverse transcription Kit (Thermo), agarose gel DNA Extraction Kit (Shanghai SANGON biological Co., Ltd.) $2 *$ Taq PCR MasterMix (Tiangen biotech Beijing Co. Ltd); /Amp medium, LB SOC medium and other commonly used reagents for domestic pure analysis. PMDC transformation vector containing double 35S promoter and Escherichia coli competent cell JM109 were preserved in our laboratory.

Methods. Extraction of total RNA from leaves using modified CTAB method. Mass detection of leaf total RNA using nucleic acid detector; primers were designed according to the FaUVR8 sequence in the GenBank database (GenBank login number: KU647690), and two primers were designed: FaUVR8-F: 5'-ACGCGTCGACATGGCAGAGGAAGCAGTTGCTGCTCCAAT-3' and FaUVR8-R: 5'- GGGGTACCGGAAGCCGTATGCGTTTCCCGTCGGTT -3', the underlined parts are Sal I and Kpn I cleavage sites, respectively. Primers were synthesized by bioengineering (Shanghai) Limited by Share Ltd. The total RNA extracted from strawberry leaves was stored at -20 cDNA by reverse transcription of Thermo's kit. FaUVR8 fragment amplification was performed using TaKaRa PrimeSTAR HS DNA Polymerase hi fi enzyme, The product was precipitated at $4{ }^{\circ} \mathrm{C}$ overnight after alcohol precipitation. Identification of over expression vector using plasmid PCR and double restriction enzyme digestion.

\section{Conclusions}

Light is one of the most important environmental factors in plants, and plays an important role in regulating the growth and development of plants. The plant through the light receptor coupled signal transduction components and transcription element network is used to sense the environment such as light intensity (impact), light (wavelength), irradiation direction and light cycle and other light signal parameters. There are four kinds of light receptors are known, phytochrome, mainly feel red and far red and blue light feeling mainly near ultraviolet region UV-A cryptochrome, mainly feel blue and green to the light and UV-B light receptors. UV-B wavelength is $290-315 \mathrm{~nm}$, energy is large, it can cause a variety of cell damage to plants, but it is also a signal transduction information. UV-B can increase the plant by environmental pressure, influence of photomorphogenesis, secondary metabolites regulation in plants (such as flavonoids, tannins and lignin biosynthesis) on plant growth and The new supersedes the old. affected.

Kliebenstein (2002) through the 1 gene mutation of Arabidopsis on Fifth chromosomes, obtained uvr8 mutant of Arabidopsis thaliana, the study found that the mutant is different from the other mutants in UV-B under the irradiation of the anthocyanin accumulation was significantly decreased, prediction of UVR8 may play an important role in signal transduction in UV-B. Rizzini (2011) was isolated from mutant Arabidopsis thaliana uvr8 and confirmed that UVR8 is the photoreceptor [6] of UV-B UVR8. As of August 2016, the number of UVR8 genes in different species registered in NCBI (National Center for Biotechnology Information) reached 769. Photoreceptors usually use cofactor as a chromophore to absorb specific wavelengths of light, but unlike UVR8, purified UVR8 does not have a non Kiev factor as a chromophore to absorb UV-B. It is speculated that UVR8 sense light may be absorbed by aromatic amino acids in protein UV-B. Gerhardt and so on (2005) confirmed that UVR8 was mainly used as the chromophore of UV-B with special amino acid residue specific Trps, and UV-B[7] was detected.

Because of the UVR8 target gene function research is not entirely in UVR8 plants, in addition to physiological function as light receptors other than remains to be improved, especially the UVR8 on the regulation of other pathogen or pest resistance related network still needs to be improved. Studies have shown that UVR8 can stimulate the biosynthesis of acid salt enhanced plant resistance to Botrytis cinerea [8], Botrytis cinerea [9] is an important disease of strawberry harm of protected cultivation, the control methods mainly rely on chemical agents, long-term use of this kind of 
medicament not only makes strawberry resistance to Botrytis cinerea, and serious pollution the environment is not conducive to agricultural products, production and sales. If the overexpression of strawberry FaUVR8 can be confirmed, the plant resistance to Botrytis cinerea can be significantly improved, which will be of great significance in the production practice. In the later stage, the constructed pMDC- FaUVR8 overexpression vector was expressed by Agrobacterium mediated genetic transformation, and the physiological function of the gene was verified. The first use of CRISPR/Cas9 fixed-point UVR8 knockout bred mutant, the constructed pMDC- FaUVR8 expression vector overexpressing plants cultivation, the two mutually control inquiry whether UVR8 can enhance resistance to Botrytis cinerea and other diseases, as well as other abiotic stress tolerance.

\section{Acknowledgements}

This work was financially supported by National Innovation Experiment Program for University Students (201510626005).

\section{References}

[1] S. Y. Wang, H. S. Lin: Antioxidant activity in fruits and leaves of blackberry, raspberry, and strawberry varies with cultivar and developmental stage. Journal of Agricultural \& Food Chemistry, Vol. 48 (2000), p.140.

[2] C. Hui, Y. Bin, and X. P. Yang et al. Anticancer activities of an anthocyanin-rich extract from black rice against breast cancer cells in vitro and in vivo. Nutr cancer, Vol. 62 (2010), p.1128.

[3] B. R. Jordan. The effects of ultraviolet-B radiation on plants: a molecular perspective. Advances in Botanical Research, Vol. 22 (1996), p. 97.

[4] D. J. Kliebenstein. Arabidopsis UVR8 regulates ultraviolet-B signal transduction and tolerance and contains sequence similarity to human regulator of chromatin condensation 1. Plant Physiology, Vol. 130(2002), p.234.

[5] L. Rizzini, J. J. Favory, and C. Cloix et al. Perception of UV-B by the Arabidopsis UVR8 Protein. Science, Vol. 332(2011), p103.

[6] K. Mao, L. Wang, and Y. Y. Li et al. Molecular Cloning and Functional Analysis of UV RESISTANCE LOCUS 8 (PeUVR8) from Populus euphratica. Plos One, Vol.10(2015), p.88.

[7] B. A. Brown, C. Cloix, G. H. Jiang et al. A UV-B-specific signaling component orchestrates plant UV protection. Proceedings of the National Academy of Sciences of the United States of America, Vol. 102(2005), p182.

[8] K. E. Gerhardt, M. I. Wilson, and B M Greenberg. Ultraviolet wavelength dependence of photomorphological and photosynthetic responses in Brassica napus and Arabidopsis thaliana. Photochemistry and Photobiology, Vol. 81(2005), p. 1061.

[9] Demkura, V. Patricia, and Ballaréet al. UVR8 Mediates UV-B-Induced Arabidopsis Defense Responses against Botrytis cinerea by Controlling Sinapate Accumulation. Molecular Plant, Vol.5(2012), p.642. 